\title{
Ophthalmic Complications of Bariatric Surgery
}

\author{
Rui Azevedo Guerreiro • Rui Ribeiro
}

(C) Springer Science+Business Media New York 2014

\begin{abstract}
Obesity is increasing vastly in the world, and the number of bariatric surgeries being performed is also increasing. Patients being submitted to bariatric surgeries, especially malabsorptive procedures, have an increased risk of developing nutrient deficiencies, which can culminate in symptomatic hypovitaminosis, if supplementation is not done correctly. The eye and the optic system need an adequate level of several vitamins and minerals to perform properly, especially vitamin $\mathrm{A}$, and this article wants to cover the main nutrients involved, the possible ophthalmic complications that can arise by their deficiency, and the management of those complications.
\end{abstract}

Keywords Bariatric surgery $\cdot$ Obesity $\cdot$ Eye $\cdot$ Postoperative complications · Vitamin A · Vitamin A deficiency

\section{Introduction}

Obesity is defined by a body mass index (BMI) over $30 \mathrm{~kg} /$ $\mathrm{m}^{2}$, and it is increasing in prevalence in the world. In 2008, estimated 1.46 billion adults globally were overweight (BMI $>25 \mathrm{~kg} / \mathrm{m}^{2}$ ) and 502 million adults were obese (BMI $>30 \mathrm{~kg} /$ $\mathrm{m}^{2}$ ). Furthermore, estimated 170 million children (aged $<18$ years) globally were classified as overweight or obese [1].

Obesity is highly associated with increased morbidity and early mortality due to the increased prevalence of chronic diseases, such as diabetes mellitus, cardiovascular disease,

R. A. Guerreiro ( $\square)$

Centro Hospitalar de Lisboa Central, EPE, Rua José António

Serrano, 1150-199 Lisbon, Portugal

e-mail: ruiazevedoguerreiro@gmail.com

R. Ribeiro

Unidade de Tratamento Cirúrgico de Obesidade e Doenças

Endócrinas, Hospital Curry Cabral, Centro Hospitalar de Lisboa

Central, EPE, Rua da Beneficência, no. 8, 1069-166 Lisbon, Portugal

e-mail: ruijsribeiro@gmail.com dyslipidemia, hypertension, nonalcoholic steatosic hepatitis, numerous cancers, musculoskeletal disorders, and other disabilities [2].

Minimal weight loss of 5 to $10 \%$ has been associated with marked reduction in morbidity and mortality, but unfortunately, traditional therapies including dietary modifications as well as medical therapy with and without psychological support are relatively ineffective to treat obesity in the long run [2].

Surgery for weight loss in obese patients is considered to be the most effective therapy [3], but it has some drawbacks. These patients after surgery have an increased risk of developing nutrient deficiencies because of vomiting, decreased food intake, food intolerance, reduction of gastric secretions, and bypass of absorption surface areas, mainly the duodenum and proximal jejunum [4].

Due to this increased risk, especially if patients are noncompliant with their vitamin and mineral supplements prescribed after surgery, they can develop ophthalmic complications because of nutrient deficiencies.

This article aims to review which bariatric surgeries are more prone to develop ophthalmic complications, which ophthalmic complications can occur and which nutrients can be involved, with a special emphasis to vitamin A deficiency (VAD).

\section{Bariatric Surgeries}

There are three different types of bariatric surgeries: restrictive, malabsorptive, and a combination of both, which means the mixed type [5].

Restrictive bariatric surgeries, which include adjustable gastric banding (AGB) (Fig. 1a) and gastric sleeve (GS) (Fig. 1b), increase the risk of a non-balanced diet, which can lead to diminished ingestion of certain vitamins and micronutrients and, ultimately, to ophthalmic complications. 


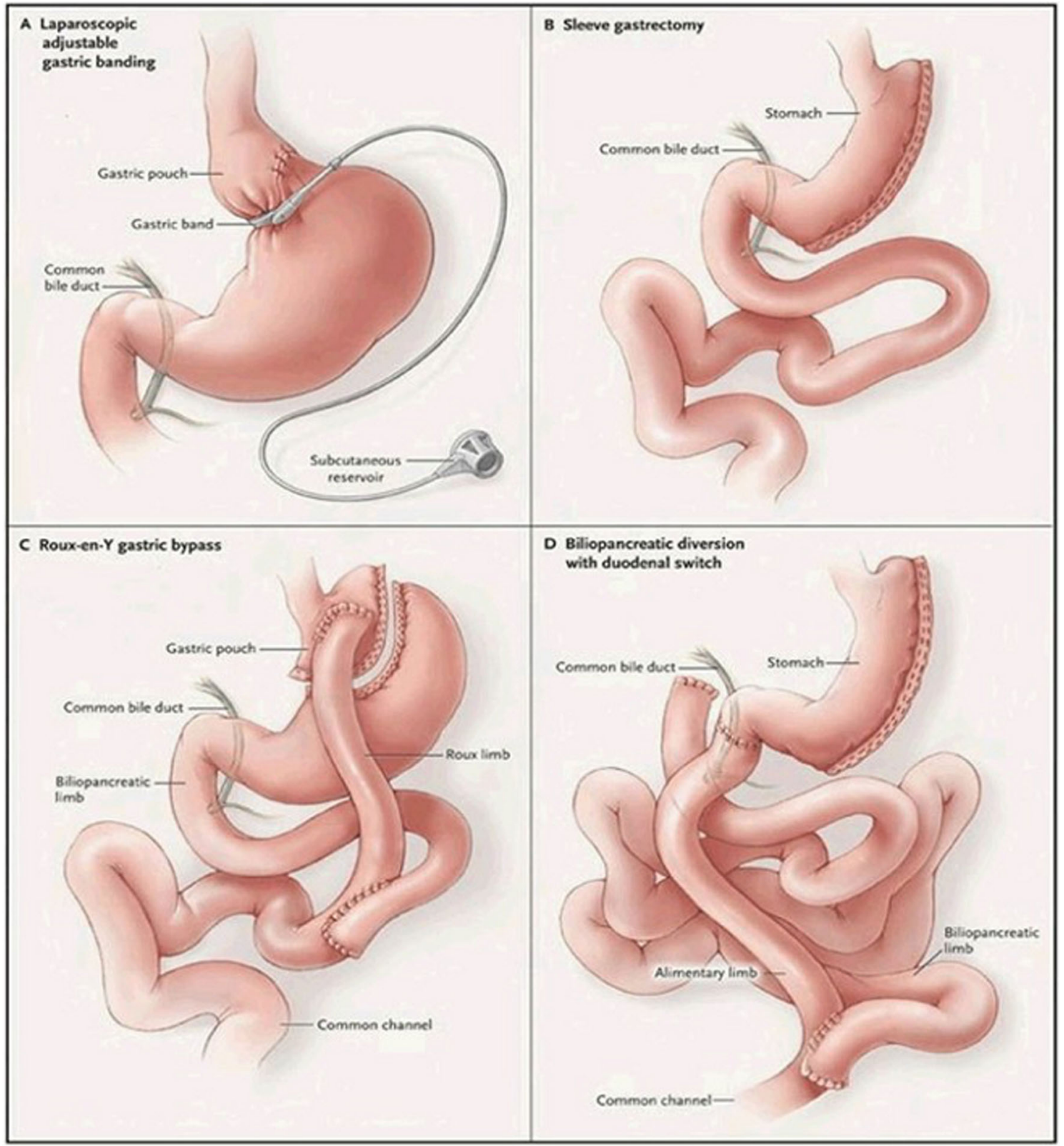

Fig. 1 Different types of bariatric surgeries [6]

On the other hand, malabsorptive and mixed bariatric surgeries, which include Roux-en-Y gastric bypass (RYGB) (Fig. 1c) and biliopancreatic diversion (BPD) (Fig. 1d), induce an iatrogenic malabsorption of vitamins and micronutrients, which can also lead to ophthalmic complications. Ophthalmic complications occur more often in malabsorptive bariatric surgeries than in restrictive or mixed bariatric surgeries [7].

\section{Ophthalmic Complications}

Bariatric surgeries can, in mid to long term, induce ophthalmic complications that can affect almost every component of the optic system: the conjunctiva, the cornea, the retina, and the optic nerve, depending from which nutrient is deficient. The main ophthalmic complications are nyctalopia (night blindness), conjunctival and corneal xerosis, corneal ulceration and scaring, keratomalacia, pigmentary retinopathy, nystagmus, ophthalmoplegia, and optic neuropathy. The overall prevalence of these complications is unfortunately unknown.

\section{Nutrients}

The literature suggests that bariatric surgery patients are at risk for deficiency of the following nutrients after surgery: vitamins $\mathrm{B}_{12}, \mathrm{~B}_{1}, \mathrm{C}$, folate, $\mathrm{A}, \mathrm{D}, \mathrm{E}$, and $\mathrm{K}$, along with the trace minerals such as iron, selenium, zinc, and copper [7]. 
From these nutrients, those whose deficiency can compromise the normal function of the optic system are vitamins A, $\mathrm{E}$, and $\mathrm{B}_{1}$ (thiamine) and copper [8].

Vitamin $\mathrm{A}$ is the main responsible for the ophthalmic complications seen after bariatric surgery, and for that reason, it will be discussed extensively in the next section.

Vitamin E deficiency can affect the retina, causing pigmentary retinopathy, but it is commonly not the presenting sign. Vitamin E deficiency usually cause a polyneuropathy, whose main signs and symptoms include areflexia, cerebellar ataxia, loss of position sense, loss of vibration sense, and muscle weakness [9].

Vitamin $\mathrm{B}_{1}$ (thiamine) deficiency can affect the eye, causing nystagmus and ophthalmoplegia, but these signs are part of a major syndrome called Wernicke's encephalopathy, which includes also ataxia and confusion.

Copper deficiency can, in rare cases, affect the optic nerve and cause an optic neuropathy $[10,11]$, but the usual presentation is a myelopathy or myeloneuropathy, which main signs and symptoms include subacute gait disorder with prominent sensory ataxia and/or spasticity [12].

Vitamin A, copper, and thiamine deficiencies and their ophthalmic complications have already occurred in patients after bariatric surgery $[11,13,14]$. Symptomatic vitamin E deficiency after gastrectomy for gastric cancer has been reported [9], but reports after bariatric surgery are lacking. Nonetheless, asymptomatic vitamin E deficiency was found in $7.1 \%$ of patients after BPD in one study [15], which means there is a potential risk for symptomatic vitamin E deficiency.

\section{Vitamin A}

\section{Metabolism}

Vitamin A can be divided into two different groups: provitamin A carotenoids, which are found in fruits and vegetables, and retinyl esters, which are found in foods of animal origin (Fig. 2).

Carotenoids are either cleaved to generate retinol or absorbed intact, but retinyl esters are completely hydrolyzed in the intestinal lumen and free retinol is taken up by enterocytes. Retinol is then reesterificated inside the enterocytes, and the resulting retinyl esters are incorporated into the chylomicrons secreted by these cells [17]. The chylomicrons are uptaken by the liver through the apolipoprotein E receptor, and this organ stores more than $90 \%$ of the body's vitamin A reserves. In healthy persons who consume an adequate diet, these reserves are sufficient to meet the body's demands for at least 6 months [16].

Retinol esters are stored in the liver and can be mobilized but, before being released, retinol binds to a specific retinolbinding protein (RBP), synthesized in the liver. The uptake of

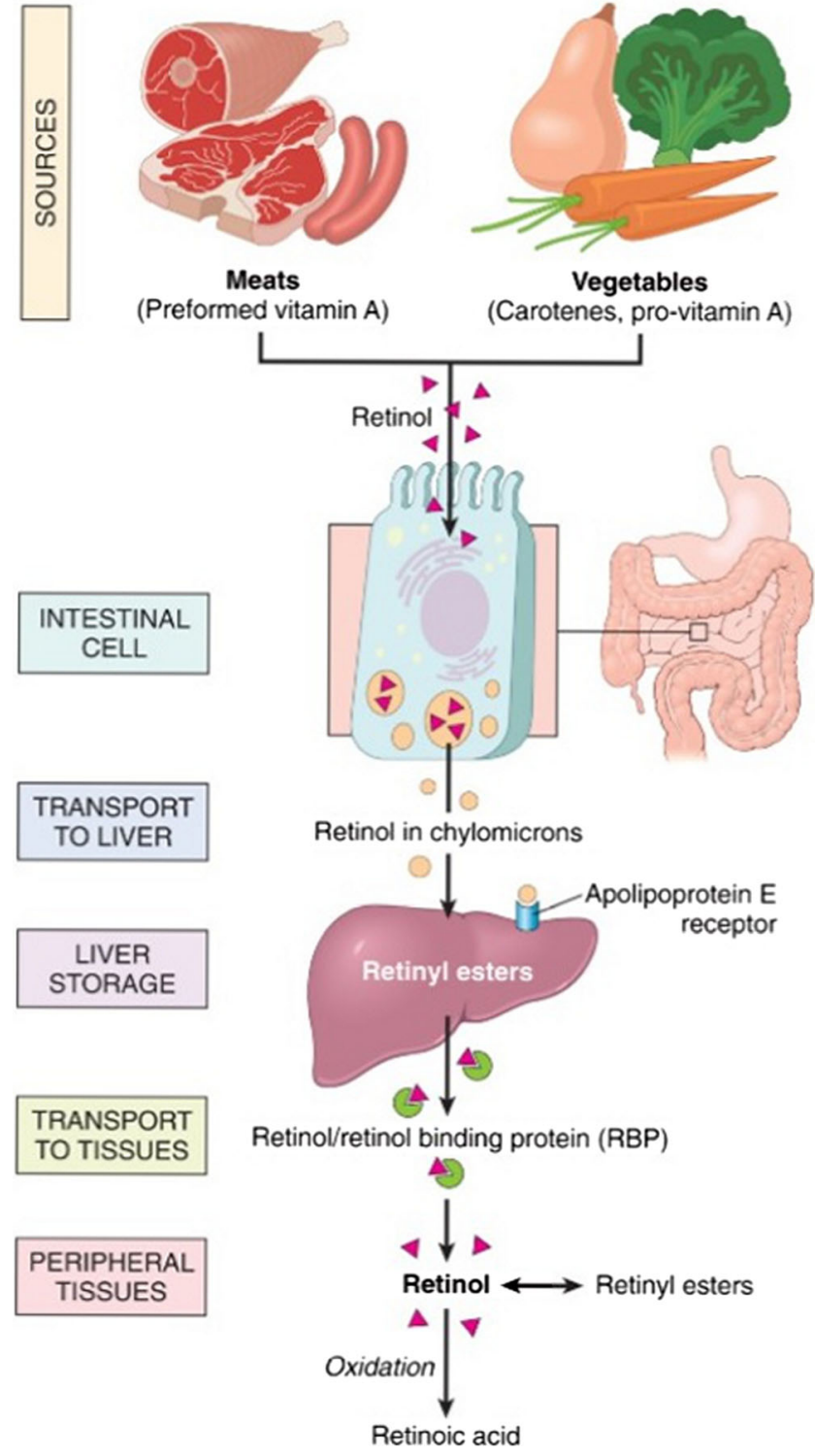

Fig. 2 Vitamin A metabolism [16]

retinol/RBP in peripheral tissues is dependent on cell surface receptors specific for RBP. After uptake, retinol binds to a cellular RBP, and the RBP is released back into the blood. Retinol may be stored in peripheral tissues as retinol ester or be oxidized to form retinoic acid [16].

Functions

Vitamin A is crucial for human life, and it has a role in reproduction, embryonic growth and development, immune competence, maintenance of epithelial surfaces, and proper functioning of the adult brain [18].

It is also vital for ocular metabolism, including maintenance of the conjunctival and corneal epithelial surfaces, retinal phototransduction, and retinal pigment epithelial viability [19]. 


\section{Vitamin A Deficiency}

Signs and Symptoms

Signs and symptoms of VAD in the eye follow a predictable sequence: nyctalopia (night blindness), conjunctival xerosis (Fig. 3) with Bitot's spots (Fig. 4), corneal xerosis (Fig. 5), corneal ulceration (Fig. 6), and localized or complete limbal to limbal corneal dissolution, known as keratomalacia (Fig. 7) [21].

\section{Epidemiology}

According to the World Health Organization, 45 countries have a public health problem at the clinical level, which includes overt signs of VAD, and 122 countries have depletive subclinical levels of vitamin A with marginal liver reserves. Although progress has been made globally to alleviate overt clinical signs of VAD, marginal vitamin A status is still prevalent and difficult to diagnose [22] (Fig. 8).

The epidemiology of VAD in patients after bariatric surgery is not well established. The results of several small studies are inconsistent, and the deficiency depends also on the type of bariatric surgery.

Regarding RYGB, a mixed bariatric surgery, Zalesin et al. [24] reported a prevalence of VAD in $35 \%$ of patients 6 weeks after RYGB and $18 \%$ of patients 1 year after surgery. Clements et al. [25] reported a prevalence of VAD in $11 \%$ of patients 1 year after RYGB and $8.3 \%$ of patients 2 years after surgery.

Regarding BPD, a more malabsorptive bariatric surgery than RYGB, the prevalence of VAD seems to be higher. Slater et al. [15] found the prevalence of VAD at $52 \%$ for patients 1 year after surgery and $69 \%$ for patients 4 years after surgery. Many of these patients reported compliance with supplements, emphasizing a possible need for parenteral supplementation of vitamin A in certain cases. Moreover, Scopinaro et al. [26] observed a $2.8 \%$ prevalence of nyctalopia in a series of patients after BPD, with no mention to ocular surface findings.

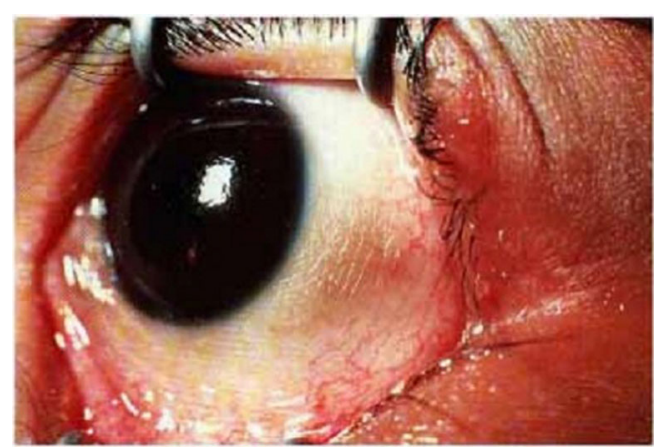

Fig. 3 Conjunctival xerosis [20]. Note dry, unwettable conjunctiva, representing keratinizing metaplasia [21]

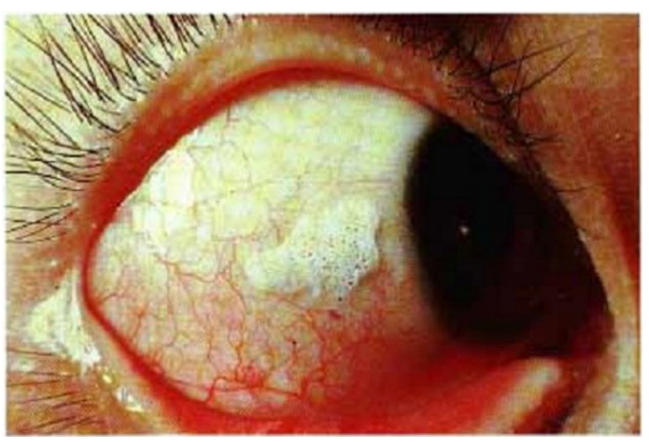

Fig. 4 Bitot's spot [20]. Bitot's spot, usually temporal (but also nasal in more advanced cases) represents desquamated keratin and an overgrowth of the xerosis bacillus (a diphtheroid) [21]

Three additional patients with nyctalopia and VAD occurring after BPD procedures were reported. One of them did not recover normal vision despite resuming vitamin A supplementation [13, 27, 28] (Fig. 9).

Regarding restrictive bariatric surgeries (AGB and GS), only a few studies and clinical reports can be found. Ledoux et al. [29] showed no statistically significant difference in prevalence of VAD between the AGB group and the control group, which might indicate that there is no increase in the risk of VAD after restrictive bariatric surgeries.

\section{Pathophysiology}

In bariatric patients, the vitamin A can be deficient duo to three different mechanisms: vitamin A metabolism impairment, malnutrition, and malabsorption [19].

Vitamin A metabolism can be impaired prior to the bariatric surgery due to the existence of nonalcoholic fatty liver disease (NAFLD), which can occur in 71 to $88 \%$ of obese patients, depending from the study [30]. NAFLD can progress from simple steatosis to nonalcoholic steatohepatitis and, ultimately, cirrhosis, and one of the mechanisms involved is an increase in oxidative stress in the liver. Vitamin A has antioxidant properties, it is stored in the liver, and it is consumed

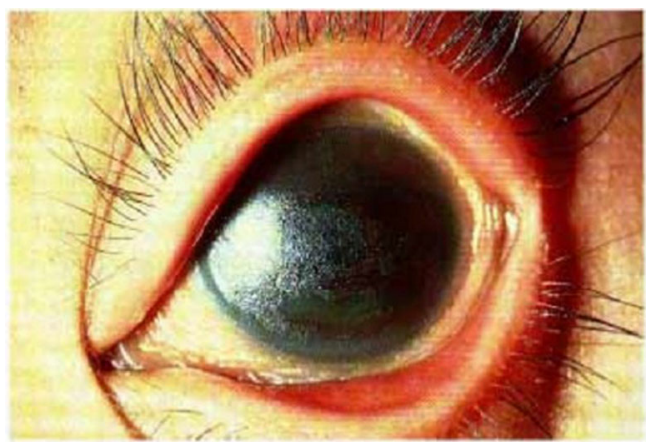

Fig. 5 Corneal xerosis [20]. Part (usually inferiorly) or the entire cornea develops a dry, lusterless appearance secondary to epithelial metaplasia [21] 


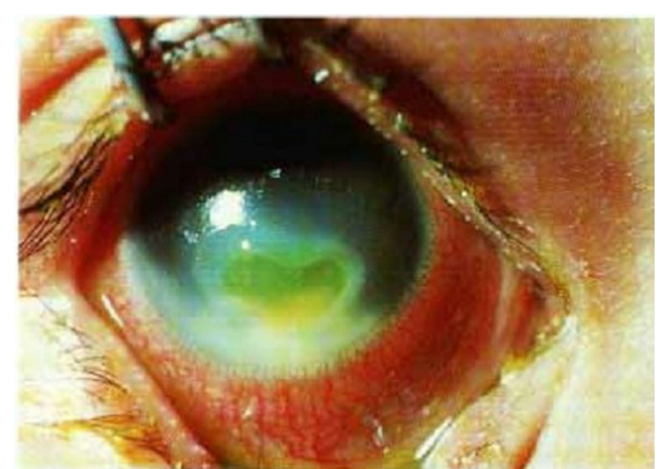

Fig. 6 Corneal ulceration [20]. Early in the course, xerophthalmic ulcers have a classical 'punched out' appearance with little if any infiltrate. In more advanced disease, secondary infection can result in a more irregular, infiltrated appearance. Most ulcers are inferior though they can appear anywhere in the periphery. Only rarely do they arise in the visual axis [21]

during the evolution of NAFLD to combat reactive oxygen species and to reduce the oxidative stress. Supporting this relationship between oxidative stress and vitamin $\mathrm{A}$ is not only the fact that serum vitamin A levels usually drop in patients with NAFLD but also the fact that the severity of NAFLD is inversely correlated with vitamin A levels in the liver [31].

Vitamin A metabolism can also be impaired by the bariatric surgery itself because the surgery constitutes an aggression to the body's homeostasis, and it increases the levels of oxidative stress in the postoperative period, which can interfere with vitamin A absorption, processing, storage, and consumption [24].

Malnutrition can occur because patients after bariatric surgery often experience drastic decreases in the dietary intake of many micronutrients, like carotenoids and retinol, especially in early recovery. In addition, traditional dietary recommendations after gastric bypass include a low-fat diet which potentially limits the absorption of fat-soluble vitamins (A, D, E, and $\mathrm{K})$ [24].

Malabsorption of nutrients is the main therapeutic mechanism of malabsorptive bariatric surgeries, but as the

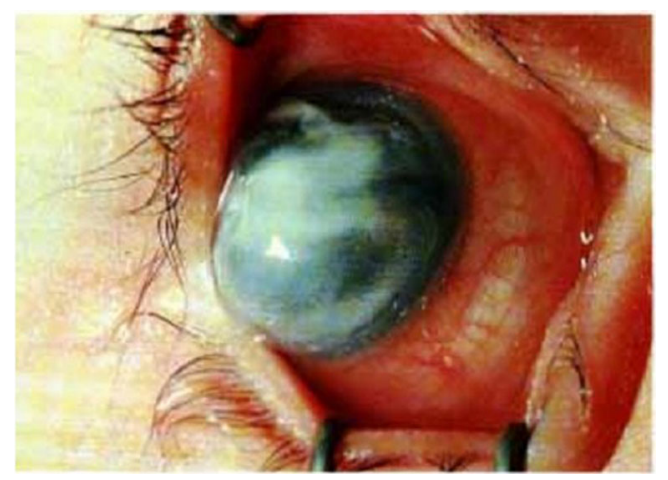

Fig. 7 Keratomalacia [20]. By the time keratomalacia presents the necrosis commonly involves the entire cornea, and the eye is ultimately lost [21] malabsorption is not selective, these surgeries create inevitably an iatrogenic malabsorption of vitamin A. This is because the food bypasses the duodenum and first portion of the jejunum, where most of the selective absorption processes take place [24].

\section{Diagnosis}

The diagnosis of VAD can be made by accessing the serum retinol concentration and/or serum RBP concentration [32].

Serum retinol is an established biochemical indicator of vitamin A status, and it is preferably assessed by highperformance liquid chromatography (HPLC). However, retinol is unstable when exposed to heat or light and HPLC, required to its quantification, is expensive. According to the American Society for Metabolic and Bariatric Surgery [33], normal levels of serum retinol are between 20 and $80 \mu \mathrm{g} / \mathrm{dL}$ and a diagnosis of VAD can be made with a concentration of serum retinol below $10 \mu \mathrm{g} / \mathrm{dL}$. Its levels can nonetheless be affected by factors that affect the release of RBP from the liver, for example, infection, protein status, adequacy of other nutrients, and organ disease [32].

Serum RBP concentration can also be used to diagnose VAD as its levels should reflect serum retinol concentration. Assessment of RBP is easier than assessment of serum retinol, cheaper, and requires less amount of serum, 10 to $20 \mu \mathrm{L}$, which can be obtained from a finger prick. However, a cutoff for serum RBP concentration has not been clearly defined and accepted. Not all RBPs found in the serum are complexed with retinol, and the binding of RBP to retinol is influenced by a number of factors such as the presence and degree of acutephase response, protein energy malnutrition, liver disease, chronic renal failure, and acute stressful situations [32]. Further studies are therefore necessary to establish serum RBP concentration as a useful marker for the diagnosis of VAD.

\section{Treatment}

The American Society for Metabolic and Bariatric Surgery (ASMBS) suggests as a treatment for VAD 10,000 to 25,000 international units (IU) of vitamin A, orally, per day, until clinical improvement (usually 1 to 2 weeks), if there are no corneal changes. In the case that cornea is already affected, the dose of vitamin A should be increased to 50,000 to 100,000 IU intramuscularly (IM) for 3 days, followed by 50,000 IU IM for 2 weeks [33].

Iron and copper deficiency should also be evaluated because their existence can impair the resolution of vitamin A deficiency [33].

Toxicity of vitamin A replacement can occur with daily doses above 50,000 IU in a period longer than 3 months. Early manifestations of toxicity include dry, scaly skin, hair loss, mouth sores, painful hyperostosis, anorexia, and vomiting. 
Fig. 8 Global prevalence of VAD in 1995 [23]

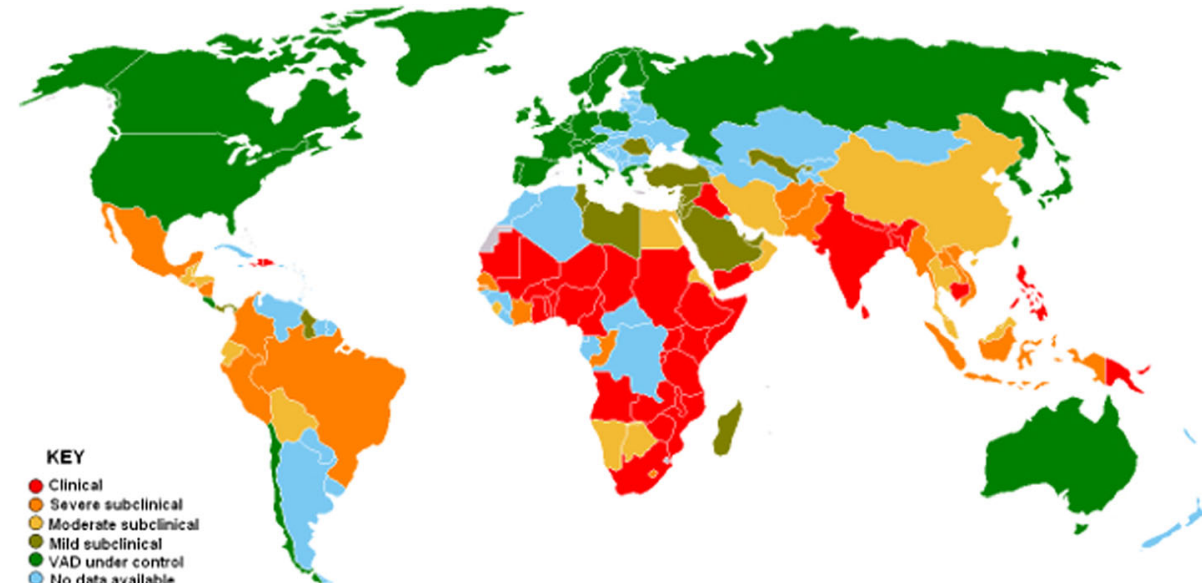

Prognosis

Night blindness is the first symptom to resolve, usually within 1 to 3 days. Conjunctival and corneal xerosis will also resolve in a couple of weeks, and none of them will lead to permanent vision-threatening sequelae. Cornea ulceration will often result in cornea scarring, and if early ulcers are usually small and peripheral, leaving the visual axis undisturbed, bigger ulcers and localized keratomalacia can rapidly spread to involve the entire cornea, leaving a blind and often painful eye [21].

This means that early diagnosis is crucial to prevent complications that are irreversible despite vitamin A replacement.

\section{Discussion}

Ophthalmic complications after bariatric surgery are apparently not frequent, but if undetected, they can have devastating consequences for the patients.

The real prevalence of these complications is unknown but the rarity of clinical reports of symptomatic nutrient deficiencies with ophthalmic complications can also mean that no one is looking for them.
Fig. 9 Prevalence of VAD found in different studies

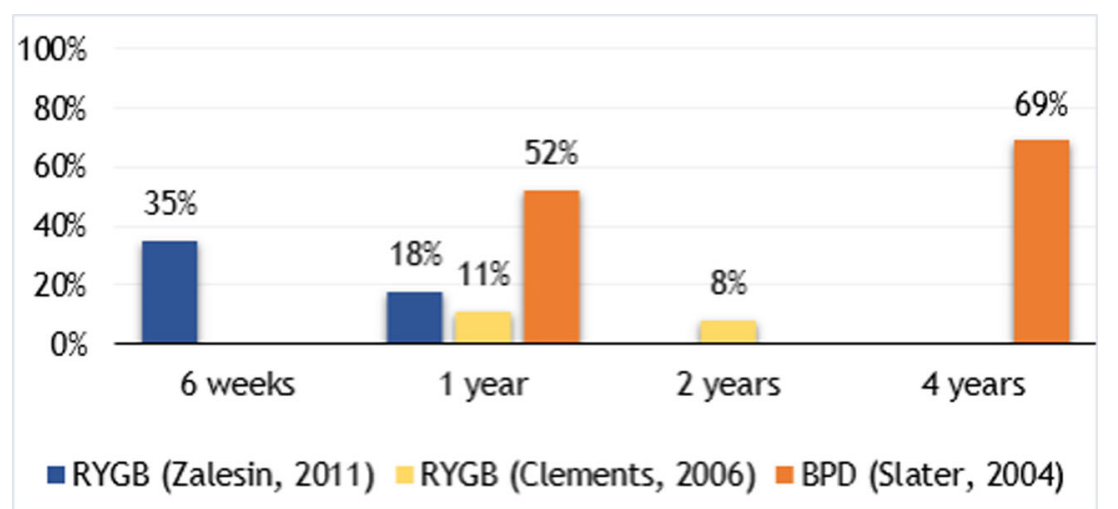


Bariatric surgery has with time been evolving to more malabsorptive than restrictive procedures, and so, the prevalence of these complications is expected to increase. Further studies are necessary to evaluate the true prevalence of these complications so that doctors and patients can be more aware of them.

Special emphasis should be done in preventing nutrient deficiencies and in increasing adherence of patients to vitamin supplementation, which in most cases, must be lifelong. If prevention could not be achieved in all patients, high clinical suspicion and early diagnosis of ophthalmic complications after bariatric surgery are crucial to prevent irreversible damages that can culminate in blindness.

Conflict of Interest The authors declare that they have no conflict of interest.

\section{References}

1. Swinburn BA, Sacks G, Hall KD, et al. The global obesity pandemic: shaped by global drivers and local environments. Lancet. 2011;378(9793):804-14.

2. Sawaya RA, Jaffe J, Friedenberg L, et al. Vitamin, mineral, and drug absorption following bariatric surgery. Curr Drug Metab. 2012;13(9): 1345-55.

3. Gloy VL, Briel M, Bhatt DL, et al. Bariatric surgery versus nonsurgical treatment for obesity: a systematic review and meta-analysis of randomised controlled trials. BMJ. 2013;347:f5934.

4. Schweiger C, Keidar A. Nutritional deficiencies in bariatric surgery patients: prevention, diagnosis and treatment. Harefuah. 2010;149(11):715-20, 48

5. Pories WJ. Bariatric surgery: risks and rewards. J Clin Endocrinol Metab. 2008;93(11 Suppl 1):S89-96.

6. DeMaria EJ. Bariatric surgery for morbid obesity. N Engl J Med. 2007;356(21):2176-83.

7. Shankar P, Boylan M, Sriram K. Micronutrient deficiencies after bariatric surgery. Nutrition. 2010;26(11-12):1031-7.

8. Parrish CR. Severe micronutrient deficiencies in RYGB patients: rare but potentially devastating. Pract Gastroenterol. 2011;100:13-27.

9. Rino Y, Yukawa N, Sato T, et al. Vitamin E deficiency begins within 6 months after gastrectomy for gastric cancer. World J Surg. 2014.

10. Naismith RT, Shepherd JB, Weihl CC, et al. Acute and bilateral blindness due to optic neuropathy associated with copper deficiency. Arch Neurol. 2009;66(8):1025-7.

11. Pineles SL, Wilson CA, Balcer LJ, et al. Combined optic neuropathy and myelopathy secondary to copper deficiency. Surv Ophthalmol. 2010;55(4):386-92.

12. Jaiser SR, Winston GP. Copper deficiency myelopathy. J Neurol. 2010;257(6):869-81.

13. Hatizifotis M, Dolan K, Newbury L, et al. Symptomatic vitamin A deficiency following biliopancreatic diversion. Obes Surg. 2003;13(4):655-7.
14. Serra A, Sechi G, Singh S, et al. Wernicke encephalopathy after obesity surgery: a systematic review. Neurology. 2007;69(6):615. author reply-6.

15. Slater GH, Ren CJ, Siegel N, et al. Serum fat-soluble vitamin deficiency and abnormal calcium metabolism after malabsorptive bariatric surgery. J Gastrointest Surg. 2004;8(1):48-55. discussion 4-5.

16. Robbins SL, Kumar V, Cotran RS. Robbins and Cotran pathologic basis of disease. 8th ed. Philadelphia: Saunders/Elsevier; 2010. p. 1450. xiv.

17. Harrison EH, Hussain MM. Mechanisms involved in the intestinal digestion and absorption of dietary vitamin A. J Nutr. 2001;131(5): 1405-8.

18. Sun H. Membrane receptors and transporters involved in the function and transport of vitamin A and its derivatives. Biochim Biophys Acta. 2012;1821(1):99-112.

19. Lee WB, Hamilton SM, Harris JP, et al. Ocular complications of hypovitaminosis A after bariatric surgery. Ophthalmology. 2005;112(6):1031-4.

20. Sommer A, World Health Organization. Vitamin A deficiency and its consequences: a field guide to detection and control. 3rd ed. Geneva: World Health Organization; 1995. p. 69. vii.

21. Sommer A. Xerophthalmia, keratomalacia and nutritional blindness. Int Ophthalmol. 1990;14(3):195-9.

22. Tanumihardjo SA. Vitamin A: biomarkers of nutrition for development. Am J Clin Nutr. 2011;94(2):658S-65.

23. World Health Organization. Global prevalence of vitamin A deficiency in populations at risk 1995-2005: WHO global database on vitamin A deficiency. Geneva: World Health Organization; 2009. p. 55.

24. Zalesin KC, Miller WM, Franklin B, et al. Vitamin a deficiency after gastric bypass surgery: an underreported postoperative complication. J Obes. 2011;2011.

25. Clements RH, Katasani VG, Palepu R, et al. Incidence of vitamin deficiency after laparoscopic Roux-en-Y gastric bypass in a university hospital setting. Am Surg. 2006;72(12):1196-202. discussion $203-4$.

26. Scopinaro N, Adami GF, Marinari GM, et al. Biliopancreatic diversion. World J Surg. 1998;22(9):936-46. PubMed PMID: 9717419.

27. Spits Y, De Laey JJ, Leroy BP. Rapid recovery of night blindness due to obesity surgery after vitamin A repletion therapy. Br J Ophthalmol. 2004;88(4):583-5.

28. Smets RM, Waeben M. Unusual combination of night blindness and optic neuropathy after biliopancreatic bypass. Bull Soc Belge Ophtalmol. 1999;271:93-6.

29. Ledoux S, Msika S, Moussa F, et al. Comparison of nutritional consequences of conventional therapy of obesity, adjustable gastric banding, and gastric bypass. Obes Surg. 2006;16(8):1041-9.

30. Angulo P. GI epidemiology: nonalcoholic fatty liver disease. Aliment Pharmacol Ther. 2007;25(8):883-9.

31. Chaves GV, Pereira SE, Saboya CJ, et al. Association between liver vitamin A reserves and severity of nonalcoholic fatty liver disease in the class III obese following bariatric surgery. Obes Surg. 2014;24(2): 219-24.

32. de Pee S, Dary O. Biochemical indicators of vitamin A deficiency: serum retinol and serum retinol binding protein. J Nutr. 2002;132(9 Suppl):2895S-901.

33. Allied Health Sciences Section Ad Hoc Nutrition Committee, Aills L, Blankenship J, et al. ASMBS allied health nutritional guidelines for the surgical weight loss patient. Surg Obes Relat Dis. 2008;4(5 Suppl):S73-108. 